\title{
Precise characterisation of isolated molecules: general discussion
}

Zlatko Bacic, David Benoit, (D) Matthieu Besemer, Joel Bowman, Stephen Bradforth, David Clary, Robert Donovan, Ingo Fischer, iD Francesco Gianturco, Majdi Hochlaf, Paul Houston, Peter Knowles, Stephen Leone, Roberto Linguerri, Uwe Manthe, Anne B. McCoy,

Jens Petersen, (D) Jeremy Richardson, Xiao Shan, Petr Slav'ıcek, Thierry Stoecklin, Krzysztof Szalewicz, Ad van der Avoird, Roland Wester, (ID Graham Worth (D) and Anne Zehnacker-Rentien

Graham Worth opened a general discussion of the Introductory Lecture by Sir David Clary: The calculations you do in general use reduced dimensionality models, hopefully extracting the physically most relevant coordinates and assuming that the other modes are spectator modes. How reasonable is this approximation for the calculations of rates? Does it depend on the timescale of the reaction, i.e. could the "spectator" modes be important for slow reactions?

David Clary replied: The reactions we study are bimolecular or unimolecular reactions with a large energy barrier. These are slow reactions at low collision energies but without major collision complexes. It is possible to evaluate which modes will be important by adding modes sequentially into the computation. This can be done in a straightforward and cost-eff ective way by using the semiclassical transition state theory and examining the magnitude of potential energy coefficients for different modes coupling with the reaction coordinate.

Graham Worth asked: How can you distinguish which are the physically important coordinates?

David Clary replied: They are the bonds being broken and formed.

Francesco Gianturco asked: One of the famous students of Aron Kupperman was Don Truhlar. He introduced a reduced dimensionality treatment of reactions which he called variational transition state theory (VTST), and it can be usefully employed to deal with exothermic reactions without a barrier that form a reaction complex. It requires knowledge of the minimum energy path and follows the reaction essentially in two dimensions. 
David Clary responded: The variational transition state theory approach with tunnelling corrections has been very successfully applied by Truhlar and co-workers to many reactions. ${ }^{1}$ There are several variants of the one-dimensional tunneling approach used in this theory, the choice of which depends essen-tially on the reaction skew angle. This is not the case in our two dimensional quantum reactive scattering approach.

1 J. L. Bao and D. G. Truhlar, Variational transition state theory: theoretical framework and recent developments, Chem. Soc. Rev., 2017, 46, 7548-7596.

Francesco Gianturco said: The variational transition state theory mentioned in my previous comments does not require a barrier and works very well for exothermic reactions which form a complex, as we have shown in some of our recent work. ${ }^{1}$

1 F. A. Gianturco, M. Satta, E. Yurtsever and R. Wester, Astrophys. J., 2017, 42, 850.

Peter Knowles asked: Is it really necessary to calculate all of the third and fourth derivatives of the potential energy surface in order to do SCTST?

David Clary answered: For bimolecular reactions it is usually possible to only consider the couplings along the reaction coordinate which gives a very powerful reduced dimensionality SCTST which only requires minimal ab initio calcula-tions. This has been demonstrated in several of our papers referenced in my introductory lecture.

Joel Bowman asked: The tunneling correction to transition state theory using semiclassical theory with a quartic force eld at the saddle point is a powerful extension of the Wigner tunneling correction, which is based on a separable normal mode model where the imaginary frequency mode describes tunneling through a locally quadratic barrier. The extension uses a non-separable theory where the action of the relevant periodic orbit is obtained using second order perturbation theory. This extension, as you and others have shown, can be lead to quite accurate tunneling corrections for simple barrier reactions. I think it would be appropriate to refer to this tunneling correction as the "Miller or Miller-Handy tunneling correction".

David Clary answered: Miller was certainly a pioneer in developing the SCTST and he, together with Handy, also did some of the rst calculations on chemical reactions with the method. However, Miller and Handy also have their names associated with other developments in theoretical chemistry and the term "semiclassical transition state theory" is more descriptive.

Stephen Leone commented: You discussed several important cases where quantum dynamics plays a signi cant role in reactive collisions, such as reactive resonances and tunneling. We also know that coherences frequently are impor-tant in short pulse laser experiments. Would you care to speculate whether we are missing some large categories of quantum effects in ordinary reactive collisions? 
David Clary answered: Another important area where quantum eff ects are important refers to problems involving couplings between more than one elec-tronically excited state. This is strictly even the case with the $\mathrm{F}+\mathrm{H}_{2}$ reaction where the spin-orbit states of the $\mathrm{F}$ atom come into play. Some papers later in the discussion meeting (e.g. the paper by Worth et al., DOI: 10.1039/C8FD00090E) are involved with quantum dynamics for several coupled electronic states. Another area is reactions at very low collision energies with very few partial waves. This is becoming an active area both experimentally and theoretically.

Majdi Hochlaf remarked: The paper and presentation of Prof. D. Clary (DOI: $10.1039 / \mathrm{C} 8 \mathrm{FD} 00131 \mathrm{~F}$ ) are dealing with processes occurring for temperatures $>200 \mathrm{~K}$. I would like to point out that quantum effects are enhanced at low tempera-tures for both reactive and non-reactive collision phenomena. Several examples can be found in the literature.

Jeremy Richardson commented: In some of your calculations, you are employing a playoff between accuracy and efficiency by computing energies using coupled cluster theory, but Hessians with MP2. Can you comment on the size of the error this introduces with respect to a full coupled cluster calculation?

David Clary responded: MP2 is generally a reliable method for calculating vibrational zero-point energies. There are many examples in the literature which include evaluating the accuracy of MP2 against $\operatorname{CCSD}(\mathrm{T})$ (see for example Merrick et al. ${ }^{1}$ ). The MP2 fundamental vibrational frequencies are normally accurate to within a few $\mathrm{cm}$ 1 . However, this accuracy is not the case for barrier heights of reactions where $\operatorname{CCSD}(\mathrm{T})$ or equivalent theory is needed.

1 Merrick et al., J Phys. Chem. A, 2007, 111, 11683.

Joel Bowman remarked: You mentioned a number of well-known quantum effects in reaction dynamics, namely tunneling and resonances. I wish to comment on a possible intriguing new quantum effect associated with the "roaming pathway" in reaction dynamics which has been investigated classically. Do you agree that this large amplitude motion, o en associated with $\mathrm{H}$ atom motion, may display interesting quantum behavior?

David Clary replied: Roaming is an interesting effect which has been been seen quite recently in both theory and experiment. As is shown nicely in your recent review, ${ }^{1}$ quasiclassical trajectories normally do well in describing the eff ect. However, there have been one or two publications which suggest quantum dynamics may also play a role (see, for example, T. Takayanagi and $\mathrm{T}$. Tanaka on the $\mathrm{H}+\mathrm{MgH}$ reaction ${ }^{2}$ ). More wavepacket simulations of roaming in chemical reactions involving $\mathrm{H}$ atoms are needed.

1 J. M. Bowman and P. L. Houston, Chem. Soc. Rev., 2017, 46, 715.

2 T. Takayanagi and T. Tanaka, Chem. Phys. Lett., 2011, 504, 130.

Anne B. McCoy opened a general discussion of the paper by Joel Bowman: Joel alluded to some comparisons of spectra obtained from quantum calculations and classical dynamics. When classical approaches are used, the main feature in the 
spectrum of $\mathrm{H}^{+}\left(\mathrm{H}_{2} \mathrm{O}\right)$ n comes out well, and other features (particularly the smaller peaks slightly lower in energy than the broad peak near $2800 \mathrm{~cm}^{1}$ ) are missing. As such approaches are o en used to model spectra of these systems, can you comment on use of these classical methods for modeling these low-temperature spectra?

Joel Bowman replied: MD simulations of the IR spectra at low temperatures are generally much better for the low frequency modes, i.e.those below roughly

$500 \mathrm{~cm}^{1}$, than for high frequency stretch modes such as $\mathrm{O}-\mathrm{H}, \mathrm{N}-\mathrm{H}$ and $\mathrm{C}-\mathrm{H}$, and certainly proton stretch modes, all of which display anharmonic and strongly anharmonic behavior typically resulting in large downshi s from MD predic-tions. A recent, relevant example showing this is in ref. 1.

1 T. K. Esser, H. Knorke, K. R. Asmis, W. Schoellkopf, Q. Yu, C. Qu, J. M. Bowman and M. Kaledin, J. Phys. Chem. Lett., 2018, 9, 798-803.

Petr Slav'1 'cek remarked: In the simulations, zero-point energy is deposited in each of the modes and the dynamics are then driven at a fully classical level. This presumably leads to zero-point energy leakage. Would some of the problems be solved by using "quantum thermostats", i.e. ensuring that the ZPE would remain with each of the modes?

Joel Bowman replied: Thanks for your question. We reported two types of molecular dynamics (MD) calculation of the dipole-dipole correlation function and from the Fourier transform of the IR spectrum. One was standard micro-canonical MD, so no zero-point energies were considered, and the other did include harmonic ZPE in each mode at $t 1 / 40$. So, your question refers to these calculations. We agree with your comment about the well-known ZPE "leak". For simulations such as ours the rate of this "leak" must be compared to the rate of the dynamical process of interest. For the IR spectrum calculation this is certainly a concern. To the best of our knowledge quantum thermostats have not been applied to MD simulations of IR spectra. It would be very interesting to try this. Other methods to prevent ZPE leak have been reported, including an active method we developed some years ago which was applied successfully to the water dimer in "A practical method to avoid zero-point leak in molecular dynamics calculations: Application to the water dimer", ${ }^{1}$ and trimer in "Zero-point energy constrained quasiclassical, classical, and exact quantum simulations of isomer-izations and radial distribution functions of the water trimer using an ab initio potential energy surface". 2 This method does not employ a thermostat and could be applied to the IR spectrum calculation.

1 G. Czak'o, A. L. Kaledin and J. M. Bowman, J. Chem. Phys., 2010, 132, 164103.

2 G. Czak'o, A. L. Kaledin and J. M. Bowman, Chem. Phys. Lett., 2010, 500, 217-222.

David Clary commented: In your calculations you also needed dipole moment surfaces to compare spectral intensities against experiment. Sometimes it is easier to get spectral frequencies right but harder to get intensities. How crucial are the dipole surfaces, or is it the potential energy surfaces that are most important for calculating the intensities? 
Joel Bowman responded: Generally we use the efficient MP2 level of theory for the dipole moment surfaces (but CCSD(T)-F12 for the PES tting). This level of theory is adequate for low-resolution, but perhaps not sufficiently accurate for line-list rovibrational intensities. For such applications we would use higher level but much more computationally demanding $\operatorname{CCSD}(\mathrm{T})$ calculations of the dipole moment.

Francesco Gianturco asked: I was wondering if you have also tried to carry out your calculations using a dipolar function of reduced dimensionality, or always employed the full-dimensionality dipolar function. In case you did, what were the effects on your nal intensity spectral distributions of such reductions?

Joel Bowman replied: We have not performed reduced dimensionality calcu-lations using the dipole moment surface. My guess is that such calculations, say using the single-mode variations of the dipole moment surface, would be quali-tatively and probably even semi-quantitatively correct.

Krzysztof Szalewicz said: While it is true that your surface for the formic acid dimer depends on 24 coordinates, it is not a complete surface in the sense that we talk about $\mathrm{N}$-dimensional surfaces for dimers. I believe your surface will not be reliable if you break the double hydrogen bond. Thus, it is a surface dependent on 24 variables, but valid only in a region of the full $24 \mathrm{D}$ space.

Joel Bowman responded: The surface does not describe the dissociation of the dimer. It does, however, describe the double proton transfer and the bound vibrational states in full dimensionality. Previous potentials were reported but with much-reduced dimensionality.

Krzysztof Szalewicz remarked: You used the term AIMD which is usually reserved for denoting on-the- y MD calculations, but I believe you really used your potential energy surface in your molecular dynamics.

Joel Bowman responded: That is correct.

Krzysztof Szalewicz asked: What would you expect to get from ring polymer MD (RPMD) calculations for your system?

Joel Bowman responded: We have performed RPMD - actually, we performed thermostatted RPMD calculations of the IR spectrum of the formic acid dimer at $300 \mathrm{~K}$ using our potential and dipole moment surfaces. The results will be re-ported shortly. In brief, what we see is a spectrum similar to the "AIMD" one but for the complex O-H and $\mathrm{C}-\mathrm{H}$ stretch bands. There is some downshi ing of those bands relative to the purely classical ones, but still not as much as is seen in the VSCF/VCI calculations. Also, the TRPMD bands are quite broad.

Krzysztof Szalewicz commented: This question was a misunderstanding of the terminology used by Professor Bowman who was referring to the method used as semiclassical while most people call RPMD a quantum method: approximate but quantum. 
Uwe Manthe asked: Coming back to Joel's work, there is a general question regarding the calculation of spectra using diff erent levels of theory. There is a hierarchy of approaches starting from the harmonic approximation, proceeding via perturbation theory and then going to anharmonic calculations using the VSCF/VCI approach. In electronic structure theory language, the VSCF/VCI approach used in Multimode calculations would be called a single reference method. However, this approach has its limits and in difficult cases multi-reference approaches are required to accurately compute vibrational levels. An example is the description of the vibrational states in protonated water clusters like the Zundel cation. While high frequency stretching excitation typically is a favorable case for the VSCF/VCI approach, the description of bending vibrations and other vibrations in the $1000 \mathrm{~cm}^{1}$ range could be more challenging. Could you comment on the limitation of the VSCF/VCI approach and when one would have to employ other approaches?

Joel Bowman responded: Excellent question. The VSCF/VCI approach taken in the code Multimode uses the Watson Hamiltonian, which has limitations for strongly curvilinear motion, such as the torsional motion of the water monomers in $\mathrm{H}_{5} \mathrm{O}_{2}{ }^{+}$. For the stretch and bend modes, the approach is usually very good. For large amplitude tunneling motion, for example in $\mathrm{H}_{3} \mathrm{O}^{+}$, this approach also works well provided the normal modes of the saddle point are used. This was demon-strated in ref. 1.

1 Q. Yu and J. M. Bowman, J. Chem. Theory Comput., 2016, 12, 5284-5292.

Ad van der Avoird remarked: Multimode can also include a single large-amplitude mode, can it not? This could be applied to include the double-proton tunneling in the formic acid dimer. Did you try this? I understood that the inclusion of a large-amplitude mode in addition to all other modes requires an adiabatic separation between the largeamplitude mode and the others. How would this work in this case?

Joel Bowman answered: Thanks for the question. The answer is "yes", and no adiabatic separation is done. The basic VSCF/VCI procedure is applied, but using the normal modes of the relevant proton (double proton in the case of formic acid dimer (FAD)) saddle point. The computational eff ort is more demanding than using the normal modes of a minimum for the following reasons. The large amplitude (imaginary frequency mode) requires a big basis to span both minima and the saddle point. Second, there is strong potential coupling to the other saddle point modes, which can also require larger bases in those modes. The strong potential coupling in FAD would be a major challenge for a sum-of-products form, as needed for example in the standard MCTDH approach. This approach has been applied successfully and most recently to the mode-speci c tunneling splittings in ref. 1.

We did not apply this large amplitude approach to the calculations of the FAD IR spectra as there is no evidence of the proton double transfer in the experi-mental spectra reported in our paper (DOI: 10.1039/C8FD00077H), which are not of sufficient resolution to resolve the expected very small splittings in the bands. The complexity of the broad $\mathrm{O}-\mathrm{H}$ stretch band is almost certainly not related in 
a major way to tunneling, but to the (related) motion of the two hydrogen bonds. Hopefully, with the availability of the potential and dipole moment surfaces, further studies of the FAD dynamics will be done.

1 Q. Yu and J. M. Bowman, J. Chem. Theory Comput., 2016, 12, 5284-5292.

Jens Petersen said: You show in your paper that the experimental IR spectrum for the formic acid dimer agrees very well with the one obtained using the VSCF/ VCI approach, while the spectrum obtained from classical MD simulations on the ab initio potential energy surface is less accurate, especially for the $\mathrm{O}-\mathrm{H}$ stretching vibration. For the MD simulations, ensembles of classical trajectories have been propagated with initial conditions pertaining to speci ed total energies. These total energies were the same for each member of a given ensemble, i.e. the set of initial conditions lies on a curve in phase space.

However, the quantum mechanical analog would be a much broader region of phase space, the classical curve would be "smeared out" and the initial conditions would correspond to a distribution of energies rather than to a single one. An example for this is the Wigner phase space distribution, which is quite o en used to generate initial conditions for MD simulations on single or multiple electronic states. Did you consider to employ such "quantum" initial conditions for the classical MD simulations and can you comment on how these might change the appearance of the simulated IR spectra?

Joel Bowman replied: Thanks for your question about using other distribu-tions of initial conditions for the classical calculations. You mentioned speci - cally the Wigner distribution, which is the quantum "phase space" distribution corresponding to the harmonic zero-point energy of each mode. As you noted, this is o en done in photodissociation calculations, and we have done this as well. There is an issue, aside from the spread in energies, with using the Wigner distribution for our calculations which propagate classical trajectories for pico-seconds on the ground electronic state. The issue is that the distribution is not stationary and so the quantum nature of the initial distribution would be "lost" as time proceeds. The resulting phase space distribution would be oscillatory and probably somewhat chaotic. Nevertheless, it might be interesting to try it.

Zlatko Bacic added: This is more of a comment than a question. Connecting experimental spectra to a Hamiltonian is also related in part to the old question of optimal coordinates, that minimize the coupling between vibrational modes, and also facilitate the assignment of the excitations. Assignment implies the existence of a zeroth-order separable Hamiltonian that in some sense is close enough to the real Hamiltonian. Therefore, vibrations that appear unassignable in one set of coordinates could conceivably be assignable in diff erent set of coordinates.

Roland Wester opened a general discussion of the paper by Roberto Linguerri: You mentioned that you expect complex, non-standard rovibronic spectra for $\mathrm{C}_{2} \mathrm{H}^{+}$. In order to aid radio-astronomical searches of this ion in the interstellar medium, one requires knowledge on its pure rotational spectrum in the micro-wave or sub-millimeter wavelength range with high resolution. Can you simulate this spectrum and what do you expect? 
Roberto Linguerri replied: Thanks for this interesting question. The full simulation of the rovibronic spectra of these complex ionic systems cannot be performed by up-todate methodologies. We would need further development of the available codes and methods.

Roland Wester asked: Do you have a way to estimate the accuracy of calculated rotational transitions for $\mathrm{C}_{2} \mathrm{H}^{+}$?

Roberto Linguerri responded: The error of the variational approach adopted in this type of computations for rovibronic levels is typically less than $0.1 \mathrm{~cm}^{1}$ (ref. 1).

1 S. Carter, N. C. Handy and R. Tarroni, Mol. Phys., 2005, 103, 6-8, 1131-1137.

Majdi Hochlaf addressed Roland Wester and Roberto Linguerri: This comment is related to the question asked by Prof. Wester about the accuracy of the computations undertaken for the $\mathrm{CCH}^{+}$cation. The nuclear motion compu-tations that we will perform to derive the rovibronic spectrum of this cation go well beyond the standard methodologies that are implemented in commercial quantum chemistry codes. Indeed, these codes are useless for such complicated cases where one needs to consider the Renner-Teller eff ect in a $3 p$ state, the spin- orbit eff ect, the anharmonicity, and the anharmonic resonances - i.e. all the couplings between the angular momenta present. For astrophysical applications, we will do the computations for total angular momentum $1 / 40,1,2, \ldots$ and the corresponding splitting is very accurate and should help for the detection of this cation in astrophysical media.

Matthieu Besemer remarked: Regarding the potential energy surface described in your talk, why did you choose the partially spin-restricted coupled cluster calculation $[\operatorname{RCCSD}(\mathrm{T})]$ instead of the spin-unrestricted coupled cluster calcula-tion [UCCSD(T)], and do you see any differences between the two methods?

Roberto Linguerri responded: In our study, we performed the coupled cluster calculations with the MOLPRO suite of ab initio programs. In Molpro, partially spinrestricted (RHF-RCCSD) and spin-unrestricted (RHF-UCCSD) coupled cluster methods are available for open-shell electronic states, both based on RHF open-shell reference functions. We decided to use the partially spin-restricted version because (1) it is known that these approaches lead to almost identical results, especially in the molecular region of the PES, (2) the RHF-RCCSD approach is signi cantly computationally more efficient than RHF-UCCSD (about 3 times faster), and (3) RHFRCCSD is virtually free from any spin-contamination.

Ad van der Avoird addressed Roberto Linguerri and Majdi Hochlaf: Did you already include the spin-orbit coupling? If you did, what is the eff ect?

Majdi Hochlaf answered: The electronic ground state of the $\mathrm{CCH}^{+}$cation is of ${ }^{3} \mathrm{P}$ symmetry. The spin-orbit constant is computed as $18 \mathrm{~cm}^{1}$. The interplay between the Renner-Teller and spin-orbit eff ects mixes the levels and leads to 
a non-trivial rovibronic pattern. We are on the way to deriving this pattern using a variational approach taking into account all angular momenta.

Roberto Linguerri replied: The calculated potential energy curves for the excited states are from MRCI/aV5Z computations that do not include the spin- orbit interaction. We evaluated the spin-orbit matrix elements between these states at speci $\mathrm{c}$ molecular geometries to gain insight into the couplings that could be important for the interpretation of the spectra of this cation. We plan to perform a variational calculation of the spin-rovibronic energy levels of the lowest triplet and singlet states of $\mathrm{C}_{2} \mathrm{H}^{+}$in the near future.

Ingo Fischer also commented on the paper by Roberto Linguerri: I would like to bring to your attention recent work on diborene, $\mathrm{HBBH},{ }^{1}$ which is an inorganic biradical and has the same number of electrons as $\mathrm{C}_{2} \mathrm{H}^{+}$. We generated it from $\mathrm{B}_{2} \mathrm{H}_{6}$ in a uorine atom discharge, recorded slow photoelectron spectra using a similar approach to that of Gans et al. ${ }^{2}$ (ref. 15 in DOI: 10.1039/C8FD00091C) and achieved excellent agreement with simulations.

Interestingly, $\mathrm{HBBH}$ has an $\mathrm{X}^{3} \mathrm{~S}_{\mathrm{g}}$ ground state, while in $\mathrm{C}_{2} \mathrm{H}^{+}$the ${ }^{3} \mathrm{~S}$ is an excited state, approximately $0.4 \mathrm{eV}$ above the $\mathrm{X}^{+}{ }^{3} \mathrm{P}$ state. Finally, in $\mathrm{C}_{2}$, which also has 12 electrons, the electronic ground state is a singlet and the $\mathrm{b}^{3} \mathrm{~S}_{\mathrm{g}}$ state is roughly $0.8 \mathrm{eV}$ higher in energy. So my question is, whether a simple picture can be obtained from your computations that explains the different ground state symmetries and the order of electronic states in $\mathrm{C}_{2} \mathrm{H}^{+}, \mathrm{C}_{2}$ and $\mathrm{HBBH}$.

1 D. Schleier, A. Humeniuk, E. Reusch, F. Holzmeier, D. Nunez-Reyes, C. Alcaraz, G. A.

Garcia, J.-C. Loison, I. Fischer, R. Mitric, J. Phys. Chem. Lett., 2018, 9, 5921.

2 B. Gans, G. A. Garcia, F. Holzmeier, J. Kruger, A. R"oder, A. Lopes, C. Fittschen, J.-C. Loison and C. Alcaraz, J. Chem. Phys., 2017, 146, 11101.

Majdi Hochlaf followed up with a nal comment: This comment is related to the question of Prof. Fischer on the possible rationalization of the nature of the ground state of 12 valence electron molecular systems. I would like to point out that this question is widely discussed in the literature (for more than 30 years). Indeed, it is connected to the identi cation and determination of the ground state of $\mathrm{C}_{2}$ diatomics, which is isoelectronic to these systems. These works showed that the nature of these molecular systems is due a subtle interplay between the outermost $p$ and $s$ orbitals. The ordering of these orbitals is extremely sensitive to the electron correlation effects.

Xiao Shan opened a general discussion of the paper by Paul Houston: It seems to me that your method is based on the harmonic oscillator model. Is it possible to include the anharmonicity in the calculation, especially for a highly anhar-monic vibrational mode such as the hindered rotors?

Paul Houston answered: The method we describe works well whatever the basis set. If you use a more complicated basis set, such as an anharmonic one, one might expect less mixing among the anharmonic states in the nal result. On the other hand, for a calculation including the intensities, one would have to calcu-late the transition dipole elements of the anharmonic states. The "double harmonic" model we use (harmonic basis functions and transition dipole 
elements between harmonic states) is probably the simplest approximation to start with.

Xiao Shan remarked: In large organic molecules, it is possible for vibrational modes corresponding to motions of atoms in completely diff erent parts of the molecule to have similar peak positions in the spectrum. They are o en not shown as well-de ned single peaks. Is it still possible to apply this method to such systems? Or maybe to analyse these types of vibration approximately based on this work?

Paul Houston answered: Thanks for your question; it is one that we have investigated. When the spectrum gets congested, there is overlap between peaks separated by the experimental linewidth. The spectrum is then not quite as "informative", and one must expect that the results will not be quite as accurate. We examined this situation by calculating the spectra that best matched the roomtemperature FAD experimental results and the jet-cooled results (see Fig. $4 \mathrm{a}$ and $4 \mathrm{~b}$ of DOI: $10.1039 / \mathrm{C} 8$ FD00075A). Of course, the jet-cooled results gave more peaks, and the $\mathrm{t}$ is likely to provide more accurate eigenvectors. Still, there was good overlap between the eigenvector results for the two spectra. Thus, the method still works; it just might not be as accurate with less "informative" spectra.

Jens Petersen asked: How unique are the solutions obtained by your approach? Is it possible to obtain, e.g., two different sets of solutions, such that the experi-mental spectrum can be equally well reproduced, but the composition of the eigenvectors in terms of basis states is different?

Paul Houston answered: Your question points to one of the features of the method. Two different solutions will not, in general, produce the same result. We investigated this issue and report some results in the second paragraph of the Discussion. Four ts gave very good reproducibility between any two sets of eigenvalues. For the eigenvectors, when comparing any pair of calculations, there was at least a $62 \%$ match when determining the most important 1,2 or 3 basis states; comparing only the most important component gave a $70 \%$ match. Thus, the detailed results are diff erent, but the general reproducibility is not too bad.

Anne B. McCoy remarked: When I think about assignments, I think about identifying a physical motion associated with a particular feature in the spectrum. Once you go through your procedure, how well are you able to make such connections and how dependent are the results on the initial set of states used in constructing the initial state space for the analysis?

Paul Houston responded: An interesting question. One can certainly calculate the motions by combining the (harmonic, in our case) basis states using the eigenvector solutions. To be honest, we have not looked carefully at these to see if anything is striking. On the other hand, there is another method for identifying some of the interesting motions. It involves making a color-coded array plot of the vector amplitudes for each eigenvalue number as a function of basis state number. A plot for the FAD is shown in Fig. S4 of the ESI (DOI: 10.1039/ 
C8FD00075A). An interesting feature of such a plot is that Fermi resonances show up as symmetrical square boxes. States 38 and 39 are in Fermi resonance, as explained in the text of the main paper (DOI: 10.1039/C8FD00075A) in the last paragraph of the Discussion.

Zlatko Bacic remarked: Fig. 4 of DOI: 10.1039/C8FD00075A shows overall good agreement between the calculated and jet-cooled experimental spectra. This makes it easy to notice a peak around $2700 \mathrm{~cm}^{1}$ in the calculated spectrum that has no experimental counterpart. Can you comment on this apparent discrepancy?

Paul Houston replied: The peak at $2700 \mathrm{~cm}^{1}$ is "struggling" to shi toward lower frequency so as to contribute to the small doublet centered on $2600 \mathrm{~cm}^{1}$. To do this, it must mix with other states that will be shi ed to higher frequency as a result of the mixing. The basis set apparently does not include sufficient state density in the region just above $2700 \mathrm{~cm}^{1}$ so that the mixing can be eff ective. Please see also my answer to Dr Benoit's question about choosing the size of the basis set.

David Clary asked: Can you predict spectra from your approach? For example, can you go from spectra to eigenvectors, modify them with masses and perhaps predict isotope eff ects for the systems of interest?

Paul Houston answered: This is an interesting question. The answer is certainly "yes", but probably not in the way that you are envisioning it. Yes, if one took the basis set and modi ed each frequency by the correct isotope effect and then reran the calculation with the new basis set, it would (probably correctly) predict the spectrum of the isotopic variant. However, one cannot in general take the eigenvectors for one isotope, modify them in some way, and then from the modi ed eigenvectors predict the new spectrum. The reason is that the mixing of states depends on their proximity to one another and to the bright states. When one substitutes a different mass, different states shi to diff erent positions, so that the original mixing, encoded in the eigenvectors, will no longer be relevant to the new molecule.

David Benoit posed a follow-up question: How do you choose the size of your basis set? Presumably, this choice is important in guiding the outcome of the assignments generated by the algorithm.

Paul Houston replied: A great question! I suppose that the one-word answer should be "carefully". In general, there will be a compromise: one wants enough states so that all the peaks in the spectrum can be accounted for, but the difficulty of the calculation increases substantially with the size of the basis set. A basis set of 42 states was used for FAD. Of course, in the "double harmonic" approxima-tion, only fundamentals can carry oscillator strength. There must be at lease one bright state. The dark states must also have the correct symmetry to be infrared active when mixed with the bright state(s). Simple combination modes are preferred over complex ones. In the end, I wrote a small program to keep track of the basis frequencies and symmetries of all combinations (to a certain, fairly low 
order), and I used it to select a basis set that had good spectral overlap coverage with the experimental spectrum.

Jens Petersen asked: How efficient is your approach in terms of computational time, compared to a full Multimode calculation, if you (a) aim to obtain a similar accuracy with both methods (b) use the same number of basis states?

Paul Houston answered: A full Multimode calculation usually requires a good potential energy surface, and that can take a graduate-student-year of time. Even a er having the PES, the calculation usually takes weeks. For FAD, the Multimode calculation used over 20 thousand basis states. The MCD method is going to be far less accurate, of course. It uses only 42 basis states. On the other hand, it does not require a PES. The whole calculation, once the basis set has been chosen, etc., takes only about half a day on a personal computer.

Ad van der Avoird remarked: In your t you start with harmonic oscillator normal mode coordinates. But in your nal $t$ of the experimental spectra the Hamiltonian and eigenstates may in fact (implicitly) relate to other, perhaps curvilinear coordinates. For eigenstates involving bend and torsional motions the coupling between such curvilinear coordinates may be less than between the rectilinear normal mode coordinates. Can you comment on that?

Paul Houston replied: You are no doubt correct. Because the basis set would be enormous if it included all torsional and bending motions, these are usually le out of the basis. The implicit assumption is that they would cause broadening of the low order combination states (to diff ering amounts, to be sure) but that the coupling among these states would be small (as you suggest). Of course, if the experimental spectrum is in the very low frequency range where these contribute, they cannot be ignored. We have little experience with this situation to date.

Anne B. McCoy asked: Motivated by David Clary's talk earlier this a ernoon, I think back to earlier studies, where it was shown that vibrational spectra could be $\mathrm{t}$ well by simple model Hamiltonians that include frequencies, anharmonicities and a limited number of resonance interactions. Such a Hamiltoninan can be used effectively to model the spectrum of for example $\mathrm{CO}_{2}$, and as in the present work, the eigenvectors will have contributions from a relatively small number of nearly degenerate states. In response to my earlier question, you mentioned that you had identi ed some small "resonance Hamiltonians" from your analysis, and I am wondering if your approach might be able to be used to construct such model Hamiltonians for, for example, formic acid dimer?

Paul Houston responded: The "resonances" I referred to in the answer to your earlier question (about identifying a physical motion associated with a particular feature in the spectrum) were Fermi resonances. In addition to these, the Hamiltonian clearly has to have mixing between the bright fundamental states and the dark combination states. Other patterns might be searched for by examining an array plot of the Hamiltonian corresponding to the nal $\mathrm{t}$ (see, for example, Fig. S6 of the ESI, DOI: $10.1039 / \mathrm{C} 8 \mathrm{FD} 00075 \mathrm{~A})$. I am not con dent that this will be a productive approach. In general, a very large number of 
Hamiltonians can provide similar eigenvectors, resulting in similarly good ts (see Section 2 of DOI: 10.1039/C8FD00075A). It will likely be difficult to observe the sort of patterns that are produced when one takes a force eld, such as that in eqn (7), and calculates a Hamiltonian.

Francesco Gianturco said: In the distant past of the 1980s, the community of people working on crossed-beam scattering theory and experiments employed a similar procedure to the one you have just discussed, in that it was de ning an "inversion problem", whereby the experimental differential cross sections for non-spherical systems (e.g. atom-molecule systems) where used at different collision energies to generate by integral equations the underlying scattering potential. However, in your case, the mapping that you do from spectra to eigenvectors does not seem to also recover a potential. So, what exactly you obtain from this inverse mapping procedure of your method?

Paul Houston replied: It works for inverse eigenvalue problems, but my understanding is that the "inversion problem" for differential cross sections is not such a problem. Section 2 of the paper (DOI: 10.1039/C8FD00075A) describes why, for systems where there are only a few components in each eigenvector that have appreciative absolute magnitude, the mapping function from Hamiltonians to eigenvectors is very favorable. It is only under these conditions that we can expect the eigenvectors to be quite accurate. This is not to say that the numerical method, suitably adjusted for the "inversion problem", would not be productive. The problem is coming up with the "suitable adjustment"!

Anne Zehnacker-Rentien opened a discussion of the paper by Ingo Fischer: You mentioned that the S0-S1 transition is completely forbidden. Could we imagine that it is slightly allowed by Herzberg-Teller coupling?

Ingo Fischer replied: The $2{ }^{1} \mathrm{Ag}\left(\mathrm{S}_{1}\right)$ state is a doubly excited state and transi-tions from the ${ }^{1} \mathrm{Ag}$ ground state are symmetry forbidden in one-photon excitation. It is imaginable that it becomes weakly allowed by coupling. For example, the $\left.2{ }^{1} \mathrm{Ag}\right) 1{ }^{1} \mathrm{Ag}$ transition of 1,3,5,7-octatetraene in octane has been observed in one-photon excitation. ${ }^{1}$ However, to the best of my knowledge, the transition has not been reported before for para-xylylene. Two-photon excitation or resonance Raman spectroscopy might be more appropriate to study the $\mathrm{S}_{1}$ state.

1 M. F. Granville, G. R. Holtom and B. E. Kohler, J. Chem. Phys., 1980, 72, 4671.

Stephen Bradforth asked: I'm really interested in understanding the chal-lenges in assigning structures in the time-dependent photoelectron spectra of relatively large molecules. You've shown the comparison of the total ion signal with theory, but does theory help assign the energy dispersed photoelectron spectra? For example, can you say whether Koopman's type correlations or calculations of the Frank-Condon band envelope can be used to assign features in the photoelectron spectra?

Ingo Fischer replied: In the photoelectron spectra recorded at diff erent delay times, as seen in Fig. 9 of DOI: 10.1039/C8FD00083B, a band at high kinetic 
energies grows with increasing delay time. It is due to ionisation from the doubly excited $S_{1}$ state that in a simple Koopman's type picture should correlate upon ionisation to the $\mathrm{D}_{1}$ state of the cation. However, we employ a multiphoton probe step, which impedes such a simple interpretation. The correlations can be modi ed by intermediate resonances and consequently be different for 3-photon and 4-photon probe steps, which are both present in the experiment. The multiphoton nature of the probe step has not been taken into account in the calculations.

Petr Slav'1 'cek remarked: The time-resolved photoelectron signal is calculated using energy criterion only - whenever the ionization is energetically open, equal probability is considered for each of the geometries. In principle, one should assume also an electronic factor (see e.g. eqn (9) in the work by Huddock et al. ${ }^{1}$ Is the assumption of equal probability for every state and geometry justi ed?

1 Huddock et al., J. Phys. Chem. A, 2007, 111, 8500-8508.

Jens Petersen answered: For the determination of energy- and angle-resolved photoelectron spectra, in principle the transition dipole moment between the initial bound state and the nal state (ionised molecule plus free electron) needs to be calculated. In the case of one-photon ionisation, this can be reduced to an integral comprising the Dyson orbital for the considered ionisation channel, the dipole moment operator, and the scattering wavefunction of the free electron. The Dyson orbital contains the information about the change of the electronic structure between the neutral and ionised molecular systems and thus corre-sponds to the electronic factor considered, e.g. in ref. 1.

In the present case of para-xylylene, the ionisation corresponds to a four-photon process, leading to a quite-involved form of the transition operator. Complete neglect of both this operator and the scattering state, while retaining only the electronic factor for the bound molecular systems, might lead to an unbalanced description of the ionisation process. Since we were only interested in a comparison of the total ionisation yields between theory and experiment, we restricted ourselves to employ the energy criterion only.

This was further motivated by previous studies of time-resolved photoelectron spectra in which both the energy criterion as well as the full transition dipole moments for ionisation were used, and good agreement of the total ionisation yields was obtained. As an example, we present in the following these quantities for the furan molecule, which were obtained from the simulated time-resolved photoelectron spectra by integrating over all energies. In ref. 2, the full transition dipole moments were employed, while in ref. 3, the energy criterion was used. The resulting time traces are presented in Fig. 1a for the full transition dipole moment calculation and in Fig. 1b for the energy criterion. In Fig. 1c the experimental photoionisation yield from ref. 3 is provided for comparison, clearly showing that the overall temporal behaviour of the signal is very similar in all three cases. This, together with the very good agreement between the theoretical and experimental data for the case of para-xylylene (Fig. 10 of DOI: $10.1039 / \mathrm{C} 8 \mathrm{FD} 00083 \mathrm{~B}$ ), indicates that application of the energy criterion for the ionisation process is justi ed for the simulation of the total ionisation yield in the present case. 


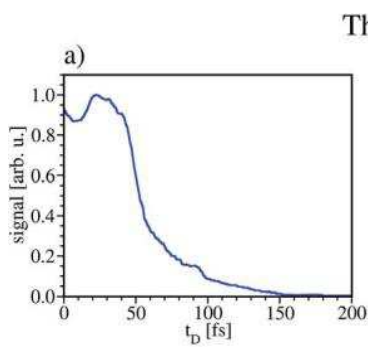

Theory

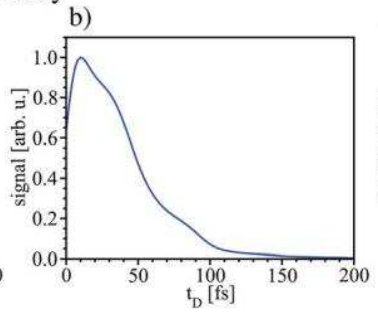

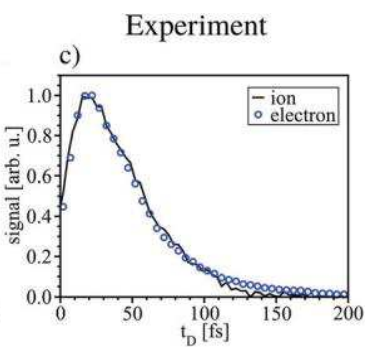

Fig. 1 Time traces from the time-resolved photoelectron spectra of the furan molecule:

(a) for the simulation using full transition dipole moments (cf. ref. 2), (b) for the simulation employing the energy criterion (cf. ref. 3) and (c) the experimental photoionisation yield from ref. 3

1 H. R. Hudock, B. G. Levine, A. L. Thompson, H. Satzger, D. Townsend, N. Gador, S. Ullrich, A. Stolow and T. J. Martinez, J. Phys. Chem. A, 2007, 111, 8500.

2 A. Humeniuk, M. Wohlgemuth and T. Suzuki, R. Mitric, J. Chem. Phys., 2013, 139, 134104.

3 T. Fuji, Y.-I. Suzuki, T. Horio, T. Suzuki, R. Mitric, U. Werner and V. Bonacic-Koutecky, J.

Chem. Phys., 2010, 133, 234303.

Petr Slav'1 'cek addressed Ingo Fischer and Jens Petersen: The non-adiabatic simulations start in the ground electronic state and the laser pulse is then switched on. This is certainly a nice approach, closely following the real experi-ment. The observed population transfer is, however, very large. The ground state depopulates by 70 percent within approximately 100 fs. Is the eld intensity realistically set in the simulations? Doesn't an interference between the ground and excited populations bring new issues/computational artifacts?

Ingo Fischer responded: In addition to the reply by Jens Petersen, I would like to point out that the laser intensities were not measured, but estimated from the beam parameters and focusing conditions. Thus, they constitute an order of magnitude estimate rather than an accurate number.

Jens Petersen replied: In our eld-induced surface hopping (FISH) simula-tions, the eld intensity corresponds to the estimated experimental value. Due to the relatively large oscillator strength of the bright $\mathrm{pp}^{*}$ state of para-xylylene, this leads to considerable population transfer. On the level of individual trajectories, subsequent cycling of the population between the ground and different excited states occurs as well, although for the whole ensemble, these eff ects are largely averaged out in the present case. However, such processes are also appropriately accounted for by the FISH method as we have previously demonstrated on the example of strong- eld quantum control in the potassium dimer, where very good agreement of the laser-induced population dynamics was obtained between quantum-classical FISH and full quantum dynamics simulations, see J. Petersen and R. Mitric. ${ }^{1}$

1 J. Petersen and R. Mitric, Phys. Chem. Chem. Phys., 2012, 14, 8299-8306.

Stephen Leone said: I would like to make a comment about how one can detect diradicals in a time-resolved experiment, such as the xylylene example of Prof. 
Fischer's paper (DOI: 10.1039/C8FD00083B). The method is X-ray spectroscopy, which is very sensitive to the radical species on particular carbon atoms. In static spectra of chloroiodomethane, all of the features can be assigned in the carbon $1 \mathrm{~s}$ and chlorine $2 p$ X-ray spectra. With the help of theoretical calculations from John Stanton, the spectroscopy of the X-ray transitions is understood. When the $\mathrm{C}-\mathrm{I}$ bond is broken in chloroiodomethane by excitation with $266 \mathrm{~nm}$ ultraviolet light, a radical is formed, leading to a singly occupied orbital on the carbon, the SOMO. This orbital is accessed with a much lower X-ray energy from the carbon $1 \mathrm{~s}$ in the X-ray and is easily distinguished in the spectrum. In contrast, the SOMO is inaccessible from the chlorine atom in the X-ray, because the transitions are very localized. Both the carbon and the chlorine X-ray transitions can probe the same $\mathrm{s}^{*}$ antibonding orbital, allowing a complete construction of the energy level diagram of the chloromethyl radical species and the orbital energy changes that occur. Both free electron laser and table top high harmonic systems are now capable of making such measurements. When the xylylene molecule would be excited electronically with $266 \mathrm{~nm}$ light, the two carbon atoms at the ends that harbor the diradical should be directly observable, and this would be the case even if the diradical character is present in the vibrationally excited molecule.

Ingo Fischer responded: The time-resolved inner-shell spectroscopy by Leone and coworkers is a wonderful technique. I would like to point out that the approach might be particularly powerful for investigating isomerisation reactions of radicals and biradicals. In this case the time-resolved ion signal carries little information and time-resolved valence shell photoelectron spectra will o en be broad. In contrast, the site-speci ty of the chemical shi that occurs in X-ray photoelectron/ESCA spectra might permit the following of isomerisation reac-tions in reactive molecules in more detail.

Robert Donovan opened a discussion of the paper by Thierry Stoecklin with a comment: The combination of $\mathrm{H}^{+}$and $\mathrm{H}$ into the lower ion-pair states of $\mathrm{H}_{2}$ could lead to characteristic emission (a signature) that could be used to identify $\mathrm{H}$ in the interstellar medium. This idea might be examined in a simple experi-ment using crossed molecular beams of $\mathrm{H}^{+}$and $\mathrm{H}$.

Thierry Stoecklin responded: This is an interesting comment. In fact, Bates considered long ago the possibility of the charge transfer reaction $\mathrm{H}^{+}+\mathrm{H} / \mathrm{H}+\mathrm{H}$ passing through excited levels of $\mathrm{H}_{2}{ }^{1}$. They found the rate of this process to be very fast, producing $\mathrm{H}$ atoms in an excited level. They concluded that the signa-ture of this process should be the rst Balmer line in the visible part of the emission spectrum. This fast and efficient process may make, in comparison, the radiative de-exciatation of $\mathrm{H}_{2}$ difficult to observe.

1 D. R. Bates and J. T. Lewis, Proc. Phys. Soc. A, 1955, 68, 173.

Francesco Gianturco remarked: I want to comment on the suggestion by Prof. Donovan on the possible detection of excited states of $\mathrm{H}_{2}$ in the form of near-Rydberg states, which could dissociate and stabilize $H$. There have been various studies by Alex Dalgarno and his group on the role of molecular hydrogen in the early universe (e.g. see ref. 1), but I have not heard them discussing the role 
of such excited states, which are usually expected to decay radiatively and therefore are not considered to be able to play a sign cant role in the formation of $\mathrm{H}$.

1 S. Lepp, P. C. Stancil and A. Dalgarno, J. Phys. B: At. Mol. Opt. Phys., 2002, 35, 57-80.

Roland Wester asked: In experiments, radiative association is usually more difficult to observe than three-body recombination. Can you estimate the three-body recombination rate coefficient for $\mathrm{H}+\mathrm{CO}+\mathrm{X}$ yielding $\mathrm{HCO}+\mathrm{X}$ ?

Thierry Stoecklin responded: It is an important point, but it is difficult to propose a value for systems including an ion as very few three-body recombination rate calculations are available. We can only refer to the recent work of Greene ${ }^{1}$ about the helium three body recombination which is about $10^{30} \mathrm{~cm}^{6} \mathrm{~s}$. This relatively large value for a rare neutral gaseous system suggests that we de nitely need to evaluate this rate for the present system.

1 J. P’erez-R’1os, S. Ragole, J. Wang and C. H. Greene, J. Chem. Phys., 2014, 140, 044307.

Roland Wester commented: The radiative association rates that you compute for $\mathrm{H}+$ CO (DOI: 10.1039/C8FD00103K) are actually comparatively high. Similar association rates have already been found experimentally, for example for the system $\mathrm{CH}_{3}{ }^{+}+\mathrm{H}_{2}$. This is very promising for future experiments.

Thierry Stoecklin replied: Thank you for this encouraging comment. We are looking forward to the rst measurements.

Francesco Gianturco remarked: I would like to mention that the quantum treatment of radiative electron attachment (REA) processes in large molecules of astrophysical interest has been carried out in the past in my group, and we found that the larger molecules had much larger REA rates than the diatomic species (e.g. $\mathrm{CN}, \mathrm{OH}$, etc.). They were reported in our publications, ref. 1 and 2, and also tested in comparison with astronomical observations in ref. 3.

1 F. Carelli, M. Satta, T. Grassi and F. A. Gianturco, Astrophys. J., 2013, 774, 97-112.

2 F. Carelli, T. Grassi, F. Sebastianelli and F. A. Gianturco, Mon. Not. R. Astron. Soc., 2013, $428,1181$.

3 F. A. Gianturco, T. Grassi and R. Wester, J. Phys. B: At. Mol. Opt. Phys., 2016, 49, 204003.

Thierry Stoecklin responded: I would like rst to answer your question about the accuracy of the REA rate coefficient by giving a few more details about the work we did which is going to be published in the Journal of Chemical Physics: "New single-center approach of photodetachment and radiative electron attach-ment: Comparison with other theoretical approaches and with experimental photodetachment data" by Thierry Stoecklin, Philippe Halvick, Jean-Christophe Loison and Miguel Lara-Moreno. In this work, we calculate the REA cross section by computing the dipole moment matrix elements between the wave function of the anion described by a Dyson orbital and the single center wave function of the scattered electron. We obtain a very good agreement with exper-imental results available for the three diatoms treated in this work. In the same work, we compare our results with those of the rst Born approximation and also 
obtain a good agreement. Concerning the work you mentioned, it is interesting, but it is based on a completely different approach as you do not calculate the dipole matrix elements needed to obtain the REA cross sections. Instead, you obtain an upper bound of the REA rate coefficient by using the statistical approach of Herbst and replacing the REA rate coefficient with the elastic one.

Francesco Gianturco remarked: In your transparencies for the model calcu-lations of the REA processes, you have shown the cross section values and the photodetachment data. Have you also obtained REA rates at low temperatures for polyatomic systems?

Thierry Stoecklin answered: Yes, we have, by simply using micro-reversibility. We agree that REA rates are larger for dipolar carbon chains of increasing size, while for non dipolar carbon chains, the increase is moderate.

Ingo Fischer opened a general discussion of the paper by Francesco Gianturco: Francesco, in Fig. 4 of your manuscript (DOI: 10.1039/C8FD00078F), photodetachment cross sections are plotted for photon energies about $80-100 \mathrm{~cm}^{1}$ lower than the electron affinity, even for temperatures of $10 \mathrm{~K}$. Here, the population of excited rotational states should be negligible. Can you comment on this plot?

Francesco Gianturco replied: The processes of populating the lower rotational levels of the molecular anions in the trap is guided by the collisions with the uploaded He gas. This occurs before the laser beam is switched on to start the photodetach-ment from those ortho/para states which are populated by the collisional mechanism. In that gure, the photodetachment process starts above the threshold of the EA of the molecule, while below that threshold the beam simply populates excited rota-tional states without photodetaching the electron. Above the threshold, however, the process is started from all the populated levels above the initial ones.

Roland Wester remarked: Negative values for the photon energy minus the electron affinity, hn EA, represent transition energies where the photodetachment process from $\mathrm{NH}_{2}$ to $\mathrm{NH}_{2}$ is driven by the combination of rotational excitation of the negative ion and photoexcitation by the photodetachment laser. Thus, the photon energy can be smaller than the electron affinity. Of course, these transitions only occur at sufficiently high rotational temperatures. One should note that in the article we do not plot the absolute transition strengths, but the ratio of the ortho and para transition strengths, which does not have to go to zero for large negative values of hn EA. At the temperatures in the ion trap, photodetachment transitions at negative values starting from 50 to $30 \mathrm{~cm}^{1}$ become relevant.

Peter Knowles commented: In understanding the shape of the potential energy surface, it is not necessary to invoke arguments involving the open-shell orbital of the neutral, since it is simpler to consider $\mathrm{NH}_{2}$ as closed-shell and isoelectronic with water. However, unlike water, the monomer electron density and electro-static potential is quite anisotropic in the angle 4, which can be explained by the greater polarisability of the non-bonding out-of-plane orbital, allowing it to expand more than the in-plane bonding orbitals in response to changing the nuclear charge from 8 to 7 . 
Francesco Gianturco answered: I thank Prof. Knowles for his interesting comment. Actually, I never linked the strong anisotropy of the $\mathrm{NH}_{2} / \mathrm{He}$ potential energy surface (PES) to the corresponding neutral with an added electron. I rather tried to point out in our work (DOI: $10.1039 / \mathrm{C} 8 \mathrm{FD} 00078 \mathrm{~F}$ ) that the presence of an extra electron in a doubly-occupied, $\mathrm{p}^{*}$-like, A1 MO, creates a negative charge distribution in the directions perpendicular, or nearly perpendicular, to the $\mathrm{N}$ atom. He is well known to have essentially repulsive interactions with negative ions or negative molecular fragments, so we expect that the spatial shape of the PES will become attractive for the molecular plane approaches and less so for the perpendicular approaches, which is what our calculations have found. It is certainly true, however, that the increased nuclear charge of the heavier atom in water will compact the spatial shape of the lone-pair and smooth out the angular dependence, as mentioned in this question.

Ad van der Avoird asked: What does the equilibrium geometry of the $\mathrm{He}-\mathrm{NH}_{2}$ complex look like? Is the $\mathrm{He}$ atom approaching in-plane, nearly perpendicular to the $\mathrm{C}_{2}$ axis of $\mathrm{NH}_{2}$ ?

Francesco Gianturco answered: We did not make a detailed structural study of the complex, e.g. its equilibrium geometry and its bound states, for, say, $\mathrm{J} 1 / 40$, since our main interest in this study was the state-changing collisional dynamics at the low relative energies of the trap. However, by analysing the overall anisotropy of the interaction potential between the anion and the He partner, we did nd that the most attractive directions of the approach were on the molecular plane and mostly on the side of the $\mathrm{N}$ atom. Our earlier paper ${ }^{1}$ shows clearly that the He atom approaching on the molecular plane from the positive direction of the $\mathrm{X}$-axis experiences the strongest attractive interaction. Thus, we could suggest that the equilibrium geometry of the complex would be along that approach, although a more quantitative analysis would be needed to get the overall minimum of the potential and its ZPE value to estimate its shallowness.

1 M. H. Vera, E. Yurtsever, R. Wester and F. A. Gianturco, J. Chem. Phys, 2018, 148, 184305.

Majdi Hochlaf asked: Is it easy to convert ortho to para (and vice versa) in the experiments, especially in the case where there are a lot of collisions?

Francesco Gianturco answered: The conversion from ortho- $\mathrm{NH}_{2}$ to para- $\mathrm{NH}_{2}$ is a process which requires the exchange of the spins of the $\mathrm{H}$ nuclei. As such, it is not governed by the electrostatic potential employed in the calculations. Thus, the calculations treat the two isomers as separate entities in the trap. From the physical standpoint, the process involving the nuclear spin occurs on a much slower timescale (typically with differences in time longer by 5-6 orders of magnitude with respect to trap uploading) hence it is practically forbidden during our trap experiments.

\section{Conflicts of interest}

There are no conflicts to declare. 